\section{Corneal collagen crosslinking for keratoconus or corneal ectasia without epithelial debridement}

N Hirji 1,2, E Sykakis ${ }^{1}$, FC Lam¹,3, R Petrarca ${ }^{1,2}$, S Hamada ${ }^{1}$ and D Lake ${ }^{1}$
${ }^{1}$ The Corneoplastic Unit, The Queen Victoria Hospital and Eye Bank, East Grinstead, UK

${ }^{2}$ Department of Ophthalmology, East

Surrey Hospital, Surrey, UK

\section{${ }^{3}$ Ophthalmology}

Department, Worthing and

St. Richards Hospital,

Western Sussex Hospitals

NHS Foundation Trust,

West Sussex, UK

Correspondence:

D Lake, The Corneoplastic

Unit and Eye Bank, The

Queen Victoria Hospital,

Holtye Road, East

Grinstead, RH19 3EB, UK

Tel: +44 (0)1342 414 560;

Fax: +44 (0)1342 414106.

E-mail: lakedamian@

hotmail.com

Received: 16 June 2014

Accepted in revised form:

16 January 2015

Published online:

20 March 2015

\begin{abstract}
Purpose Corneal collagen crosslinking (CXL) is a relatively new technique to reduce the progression of keratoconus. The technique can be performed with or without complete debridement of the corneal epithelium. We describe a novel intermediate technique involving mechanical disruption of the epithelium, and evaluate its safety and efficacy.

Methods The case notes of 128 eyes with progressive keratoconus or iatrogenic corneal ectasia who had undergone CXL using the epithelial disruption technique were retrospectively reviewed. Thin corneas were treated with hypotonic riboflavin. All others were treated with an isotonic solution. Note was made of preoperative and postoperative parameters, including uncorrected visual acuity (UCVA), best spectacle-corrected visual acuity (BSCVA), refraction, endothelial cell count, and corneal tomography. Occurrence of procedure-related complications was recorded. Statistical analyses were performed using the paired sample $t$-test and Wilcoxon signedrank test, with a level of $P<0.05$ being accepted as statistically significant. Results At 12 months, $41.8 \%$ of patients treated with isotonic riboflavin had improved UCVA and $29.7 \%$ had improved BSCVA. Only $13.4 \%$ lost lines of UCVA and $14.9 \%$ lost BSCVA. Of the patients treated with hypotonic riboflavin, at 12 months, 75\% demonstrated stability of BSCVA and $25 \%$ had stable Kmax. In addition, $25 \%$ showed improved visual acuity at $\mathbf{1 2}$ months, and $58.3 \%$ showed regression of their Kmax. Our rate of short-term complications was comparable to studies using complete epithelial removal.

Conclusions CXL with epithelial disruption is a safe and effective treatment for keratoconus or iatrogenic corneal ectasia,
\end{abstract}

and may be better tolerated by patients than the epithelium-off technique.

Eye (2015) 29, 764-768; doi:10.1038/eye.2015.23; published online 20 March 2015

\section{Introduction}

Corneal ectasia is associated with progressive corneal steepening and thinning. Problems may range from relatively mild irregular astigmatism that may be corrected by rigid contact lenses to severe visual disability requiring keratoplasty. 1,2

Corneal ectasia may be caused by keratoconus or may be iatrogenic after laser vision correction among other causes, but the common final pathway is a loss of integrity of the corneal structure.

Corneal collagen cross-linking (CXL) is a relatively new treatment to reduce or even stop the progression of ectasia. ${ }^{3}$ Developed in Dresden in the 1990s, the technique is based on the principle that corneal tissue affected by ectasia shows biomechanical weakness compared with normal corneal tissue. ${ }^{4} \mathrm{CXL}$ involves photopolymerization of collagen fibres within the corneal stroma. Riboflavin (a photosensitizing substance) and ultraviolet A (UVA) light react to produce free radicals that act on collagen side chains to increase the number of intra- and interfibrillar covalent bonds within the stroma, increasing the corneal rigidity. ${ }^{5}$ The crosslinked fibres form a dense meshwork, increasing the overall stability of the cornea. The technique has been shown to be safe, with maintenance of corneal transparency, no endothelial damage (as long as the corneal stromal thickness is $>400 \mu \mathrm{m}$ ), and no damage to more posterior structures within the eye. ${ }^{6}$

As riboflavin is a hydrophilic compound, studies have suggested that CXL should be performed following debridement of the central corneal epithelium to enable the riboflavin to efficiently permeate through to the corneal 
stroma. ${ }^{7}$ However, epithelial debridement causes patients significant pain and visual disturbance in the immediate postoperative period, until the epithelium heals.

Furthermore, stromal haze may develop in the initial few weeks or months following this conventional method of CXL, causing transient reduction in vision. ${ }^{8}$ In view of these factors, some clinicians have performed CXL without debridement of the epithelium (transepithelial CXL). Other studies have questioned whether any riboflavin diffuses into the corneal stroma through an intact epithelium, ${ }^{9}$ and questions have been raised about the efficacy of the transepithelial technique in patients with more severe keratoconus. ${ }^{10}$ There seems, therefore, a place for a CXL technique that involves minimal disturbance to the corneal epithelium, but where efficacy and safety are maintained for even the most severe cases of keratoconus. ${ }^{11}$ In an attempt to address this, a hybrid technique for CXL has been adopted at The Corneoplastic Unit at Queen Victoria Hospital, East Grinstead (UK). The technique involves disruption of the corneal epithelium using a custom-designed surgical instrument, in place of the complete epithelial debridement performed during conventional CXL. ${ }^{11}$ The technique of CXL using epithelial disruption was first developed and introduced in our department in 2007 by Dr Sheraz Daya, and we report our results to date.

\section{Materials and methods}

To date, 128 eyes with progressive keratoconus or iatrogenic corneal ectasia have undergone CXL using the epithelial disruption technique at The Corneoplastic Unit, Queen Victoria Hospital, East Grinstead (UK). The case notes of these patients were analysed retrospectively. In all cases, riboflavin/UVA CXL was performed using the CBM VEGA X-Linking system (CSO, Firenze, Italy).

All statistical analyses were performed with SPSS for Windows version 17.0 (PASW, Chicago, IL, USA). Paired sample $t$-test and Wilcoxon signed-rank test were used. All data are expressed as mean \pm SD. A level of $P<0.05$ was accepted as statistically significant.

\section{CXL technique}

Each patient initially had 4 drops of Ofloxacin $0.3 \%$ and 2 drops of Pilocarpine $2 \%$ instilled into the eye to be treated. Topical anaesthesia of the cornea was then achieved by instilling benoxinate into the eye every $5 \mathrm{~min}$. After inserting the eyelid speculum, the corneal epithelium over the complete corneal surface was disrupted using an Epithelial Disruptor (Duckworth and Kent, Hertfordshire, UK). A solution of isotonic riboflavin $0.1 \%$ (Ricrolin) with dextran was applied to the corneal surface every $3 \mathrm{~min}$ for a minimum total of $30 \mathrm{~min}$, and continued until riboflavin was observed within the anterior chamber with slit-lamp biomicroscopy using cobalt blue light. The eye was subsequently irradiated for $30 \mathrm{~min}$, with UVA at a working distance of $54 \mathrm{~mm}$ and an irradiance of $3 \mathrm{~mW} / \mathrm{cm}^{2}$ using a VEGA CBM X-Linker (CSO). During this time, riboflavin $0.1 \%$ (Ricrolin) with dextran was applied to the eye every $5 \mathrm{~min}$.

\section{CXL technique for thin corneas}

For those patients with thin corneas, hypotonic riboflavin $0.1 \%$ without dextran was used in place of the isotonic solution. In these patients, corneal thickness was re-measured before irradiation, and crosslinking was commenced when a minimum central corneal thickness of $400 \mu \mathrm{m}$ was reached.

In all patients, a bandage contact lens was inserted at the end of the procedure, and patients were medicated with chloramphenicol q.d.s. for 10 days.

\section{Follow-up}

Patients were reviewed at 5 days, and the bandage contact lens was removed at this visit. Patients were subsequently reviewed at a range of intervals following CXL. Preoperative and follow-up examinations included: uncorrected visual acuity (UCVA) and best spectaclecorrected visual acuity (BSCVA) measured on a LogMAR scale, refraction, slit-lamp biomicroscopy (Haag Streit, Mason, OH, USA), endothelial cell count (Tomey EM-3000 Specular Microscope, Phoenix, AZ, USA), and corneal tomography (Pentacam HD, Arlington, WA, USA). Note was made of any procedure-related complications that occurred.

\section{Results}

In total, data were analysed from 127 eyes of 103 patients. Out of these 127 eyes, 112 had been diagnosed with progressive keratoconus and 15 with iatrogenic ectasia. A total of 112 eyes of 89 patients (71 males and 18 females) were treated with isotonic riboflavin $0.1 \%$ (Ricrolin) with dextran. The mean age of these patients was 29.8 years (range: 19-59 years). Follow-up data at 9 and 12 months were available for 98 eyes and 84 eyes, respectively. For these patients treated with isotonic riboflavin, mean preoperative Kmax (highest $\mathrm{K}$ on Pentacam corneal tomography) was $57.6 \mathrm{D}( \pm 7.81 \mathrm{D}$ SD) that reduced to $56.3 \pm 6.48 \mathrm{D}$ SD at 12 months $(P=0.037)$. Mean endothelial cell count (ECC) was 2588.5 cells $/ \mathrm{mm}^{2}\left( \pm 282.40\right.$ cells $\left./ \mathrm{mm}^{2} \mathrm{SD}\right)$ preoperatively and 2507.3 cells $/ \mathrm{mm}^{2}\left( \pm 323.37\right.$ cells $/ \mathrm{mm}^{2}$ $\mathrm{SD})$ postoperatively at 12 months $(P=0.06)$. Mean thinnest point pachymetry (TPP) was $450.6 \mu \mathrm{m}$ 
$( \pm 40.84 \mu \mathrm{m}$ SD) preoperatively and $453.7 \mu \mathrm{m}( \pm 46.66 \mu \mathrm{m}$ SD) postoperatively $(P=0.41)$. Table 1 shows the changes in LogMAR UCVA and BSCVA in these patients at 9 and 12 months. Table 2 shows changes in Kmax and Kmean (average central K on Pentacam corneal tomography) at 9 and 12 months.

Two eyes treated with isotonic riboflavin developed microbial keratitis after procedure, 4 developed sterile infiltrates, and 6 developed mild transient stromal haze.

In all, 15 eyes of 14 patients (12 males and 2 females) were treated with hypotonic riboflavin $0.1 \%$ without dextran. The mean age of these patients was 26.7 years (range: 13-46 years). Follow-up data at 9 and 12 months were available for 3 eyes and 12 eyes, respectively. For these patients treated with hypotonic riboflavin, mean preoperative Kmax was $65.24 \mathrm{D}( \pm 8.6 \mathrm{D} \mathrm{SD})$ that reduced to $63.7 \pm 7.5 \mathrm{D}$ SD at 12 months $(P=0.137)$. Mean ECC was 2583.4 cells $/ \mathrm{mm}^{2}\left( \pm 233.75\right.$ cells $\left./ \mathrm{mm}^{2} \mathrm{SD}\right)$ preoperatively and 2458.0 cells $/ \mathrm{mm}^{2}\left( \pm 281.99\right.$ cells $\left./ \mathrm{mm}^{2} \mathrm{SD}\right)$

postoperatively $(P=0.9)$. Mean TPP was $416.8 \mu \mathrm{m}$ $( \pm 27.11 \mu \mathrm{m}$ SD) preoperatively and $419.2 \mu \mathrm{m}( \pm 33.01 \mu \mathrm{m}$ SD) postoperatively $(P=0.38)$. Table 1 shows the changes in LogMAR UCVA and BSCVA in these patients at 9 and 12 months. Table 2 shows changes in Kmax and Kmean at 9 and 12 months.

No complications were noted in this group of patients.

Table 3 summarizes the change from baseline in UCVA, BSCVA, Kmax, Kmean, ECC, and TPP at 12 months postoperatively in both the hypotonic and isotonic groups.

\section{Discussion}

CXL has been a revolutionary treatment in the management of corneal ectasia. Previous management of ectasia addressed the sequelae of ectasia such as irregular astigmatism, scarring, and poor vision with contact lenses, intracorneal rings, and keratoplasty (deep lamellar and penetrating), but was unable to effect change at the cellular level and prevent disease progression. As evidence of the efficacy and safety of collagen crosslinking has been published in the literature, its adoption by the ophthalmic community and health-care systems (including NICE in the United Kingdom) has increased.

The original Dresden protocol described complete epithelial removal within a $9 \mathrm{~mm}$ zone to enable the large riboflavin molecule to penetrate directly into the corneal stroma. Some investigators have proposed not removing the epithelium so as to prevent pain after the procedure that is unpopular with patients.

Our protocol is a hybrid method, with perforations created in the epithelium over a 9-mm zone. The perforations allow direct access for the riboflavin to the corneal stroma, but require a longer immersion time with riboflavin (often over $60 \mathrm{~min}$ ).

Following the introduction of the described modified method, we have performed a small pilot comparative study (E Sykakis and D Lake, unpublished data), comparing the tolerance and overall patient experience with the epithelium-off technique and the hybrid one in the fellow eye, with patients expressing a significant preference for the second method.

Table 1 Table demonstrating proportion of patients (\%) treated with isotonic or hypotonic riboflavin whose LogMAR UCVA and BSCVA either improved, deteriorated, or remained stable at 9 and 12 months from baseline

\begin{tabular}{|c|c|c|c|c|c|c|c|c|}
\hline & \multicolumn{4}{|c|}{ Isotonic } & \multicolumn{4}{|c|}{ Hypotonic } \\
\hline & \multicolumn{2}{|c|}{9 Months } & \multicolumn{2}{|c|}{12 Months } & \multicolumn{2}{|c|}{9 Months } & \multicolumn{2}{|c|}{12 Months } \\
\hline & UCVA & $B S C V A$ & UCVA & $B S C V A$ & UCVA & $B S C V A$ & UCVA & $B S C V A$ \\
\hline Improved by 2 lines or more & 39.0 & 52.9 & 41.8 & 29.7 & 0 & 33.3 & 25.0 & 25.0 \\
\hline Deteriorated by 2 lines or more & 24.4 & 14.7 & 13.4 & 14.9 & 33.3 & 0.0 & 8.3 & 0.0 \\
\hline Remained stable (within 2 lines of baseline) & 36.6 & 32.4 & 44.8 & 55.4 & 66.7 & 66.7 & 66.7 & 75.0 \\
\hline
\end{tabular}

Table 2 Table demonstrating proportion of patients (\%) treated with isotonic or hypotonic riboflavin in whom Kmax and Kmean regressed, progressed, or remained stable at 9 and 12 months from baseline

\begin{tabular}{|c|c|c|c|c|c|c|c|c|}
\hline & \multicolumn{4}{|c|}{ Isotonic } & \multicolumn{4}{|c|}{ Hypotonic } \\
\hline & \multicolumn{2}{|c|}{9 Months } & \multicolumn{2}{|c|}{12 Months } & \multicolumn{2}{|c|}{9 Months } & \multicolumn{2}{|c|}{12 Months } \\
\hline & Kmax & Kmean & Kmax & Kmean & Kmax & Kmean & Kmax & Kmean \\
\hline Regressed by 1D or more & 40.5 & 19.6 & 39.7 & 15.3 & 66.7 & 33.3 & 58.3 & 16.7 \\
\hline Progressed by 1D or more & 24.3 & 13.0 & 15.9 & 18.8 & 33.3 & 33.3 & 16.7 & 25.0 \\
\hline Remained stable (progressed or regressed by less than 1D) & 35.2 & 67.4 & 44.4 & 65.9 & 0.0 & 33.3 & 25.0 & 58.3 \\
\hline
\end{tabular}


Table 3 Table summarizing the change from baseline in UCVA, BSCVA, Kmax, Kmean, ECC, and TPP at 12 months postoperatively in both the hypotonic and isotonic groups

\begin{tabular}{|c|c|c|c|c|}
\hline \multirow[t]{2}{*}{ Parameter } & \multicolumn{2}{|c|}{ Isotonic } & \multicolumn{2}{|c|}{ Hypotonic } \\
\hline & Pre-op & 12 Months post-op & Pre-op & 12 Months post-op \\
\hline $\mathrm{UCVA}( \pm \mathrm{SD})$ & $0.8( \pm 0.44)$ & $0.7( \pm 0.45)$ & $0.6( \pm 0.14)$ & $0.6( \pm 0.34)$ \\
\hline $\mathrm{BSCVA}( \pm \mathrm{SD})$ & $0.3( \pm 0.27)$ & $0.2( \pm 0.20)$ & $0.3( \pm 0.21)$ & $0.2( \pm 0.13)$ \\
\hline $\mathrm{Kmax}( \pm \mathrm{SD})$ & $57.6 \mathrm{D}( \pm 7.81)$ & $56.3 \mathrm{D}( \pm 6.48)$ & $65.24 \mathrm{D}( \pm 8.6)$ & $63.7 \mathrm{D}( \pm 7.5)$ \\
\hline Kmean $( \pm S D)$ & $48.1 \mathrm{D}( \pm 4.55)$ & $47.8 \mathrm{D}( \pm 3.93)$ & $51.2 \mathrm{D}( \pm 4.36)$ & $52.6 \mathrm{D}( \pm 4.51)$ \\
\hline $\mathrm{ECC}( \pm \mathrm{SD})$ & 2588.5 cells $/ \mathrm{mm}^{2}( \pm 282.40)$ & 2507.3 cells $/ \mathrm{mm}^{2}( \pm 323.37)$ & 2583.4 cells $/ \mathrm{mm}^{2}( \pm 233.75)$ & 2458.0 cells $/ \mathrm{mm}^{2}( \pm 281.99)$ \\
\hline $\mathrm{TPP}( \pm \mathrm{SD})$ & $450.6 \mu \mathrm{m}( \pm 40.84)$ & $453.7 \mu \mathrm{m}( \pm 46.66)$ & $416.8 \mu \mathrm{m}( \pm 27.11 \mu \mathrm{m})$ & $419.2 \mu \mathrm{m}( \pm 33.01)$ \\
\hline
\end{tabular}

Studies by Samaras et al ${ }^{12}$ have demonstrated riboflavin absorption into the stroma after corneal epithelial disruption in a similar manner, but this study was limited as the riboflavin was only applied for $35 \mathrm{~min}$. This is the minimum time of immersion for our patients, and riboflavin is often applied for over $60 \mathrm{~min}$ to achieve adequate riboflavin staining of the stroma and penetration into the anterior chamber.

Rechichi et al ${ }^{11}$ performed a prospective controlled study using the same method in 28 eyes using the isotonic riboflavin only in eyes with minimum corneal thickness of $400 \mu \mathrm{m}$ and their results showed a similar improvement in mean UCVA and BSCVA at 12 months. Our results for isotonic riboflavin show that at 12 months, $41.8 \%$ of patients had improved UCVA and $29.7 \%$ had improved BSCVA, whereas only $13.4 \%$ lost lines of UCVA and $14.9 \%$ lost BSCVA. The smaller study by Rechichi et al ${ }^{11}$ also demonstrated improvement in the keratometry of treated eyes at 12 months; although modest improvement was demonstrated compared with our treated cohort, a direct comparison with our data cannot be performed ${ }^{13}$ as a Placido-disk topographer was used in their study.

Raiskup and Spoerl ${ }^{14}$ using an epithelium-off technique on 32 eyes, and $0.1 \%$ hypoosmolar riboflavin for corneas thinner than $400 \mu \mathrm{m}$ demonstrated stable mean maximum keratometry reading at the apex of $65.6 \pm 11.2 \mathrm{D}$ preoperatively and $64.9 \pm 11.0 \mathrm{D}$ postoperatively, with a similar stability in the mean BCVA (0.63 LogMAR preoperatively and 0.59 LogMAR postoperatively). Our results with hypotonic riboflavin with corneas thinner than $400 \mu \mathrm{m}$ demonstrated stability of BSCVA in $75 \%$ of patients, and $25 \%$ had stable Kmax at 12 months. Also, $25 \%$ of patients actually showed improved visual acuity at 12 months, and $58.3 \%$ showed regression of their Kmax.

Safety is a primary concern of any newly introduced procedure, and the rate of short-term complications such as infectious bacterial keratitis in our hybrid technique has been shown to be comparable to other studies with complete epithelial removal. In our series, 2 patients from $128(1.56 \%)$ developed bacterial keratitis that improved with appropriate antibiotic therapy. No patient developed herpes simplex or acanthamoeba keratitis that has been described in other case series. ${ }^{15,16}$

\section{Conclusion}

CXL with epithelial disruption is a safe and effective treatment for corneal ectasia, provided sufficient time is allowed for diffusion of riboflavin into the stroma and corneal stromal thickness is respected.

\section{Summary}

What was known before

- Corneal collagen crosslinking is a relatively new treatment to slow or halt the progression of corneal ectasia, such as keratoconus.

- It typically involves complete debridement of the central corneal epithelium that can result in significant pain and visual disturbance postoperatively.

What this study adds

- We describe a novel technique of corneal collagen crosslinking that requires disruption of the corneal epithelium as opposed to total debridement.

- Our results show this technique has comparable safety and efficacy to the traditional method, and may be better tolerated by patients.

\section{Conflict of interest}

The authors declare no conflict of interest.

\section{Acknowledgements}

We acknowledge Mr Sheraz Daya who first described the technique of corneal collagen crosslinking using epithelial disruption.

\section{References}

1 Grewal DS, Brar GS, Jain R, Sood V, Singla M, Grewal SP. Corneal collagen crosslinking using riboflavin and ultraviolet-A light for keratoconus: one-year analysis using 
Scheimpflug imaging. J Cataract Refract Surg 2009; 35(3): 425-432.

2 Hersh PS, Greenstein SA, Fry KL. Corneal collagen crosslinking for keratoconus and corneal ectasia: one-year results. J Cataract Refract Surg 2011; 37(1): 149-160.

3 Wollensak G, Spoerl E, Seiler T. Riboflavin/ultraviolet-Ainduced collagen crosslinking for the treatment of keratoconus. Am J Ophthalmol 2003; 135: 620-627.

4 Andreasson TT, Simonsen AH, Oxlund H. Biomechanical properties of keratoconus and normal corneas. Exp Eye Res 1980; 31: 435-441.

5 Caporossi A, Mazzotta C, Baiocchi S, Caporossi T. Long-term results of riboflavin ultraviolet a corneal collagen cross-linking for keratoconus in Italy: the Siena eye cross study. Am J Ophthalmol 2010; 149(4): 585-593.

6 Hayes S, O'Brart DP, Lamdin LS, Doutch J, Samaras K, Marshall J et al. Effect of complete epithelial debridement before riboflavin-ultraviolet-A corneal collagen crosslinking therapy. J Cataract Refract Surg 2008; 34(4): 657-661.

7 Baiocchi S, Mazzotta C, Cerretani D, Caporossi T, Caporossi A. Corneal crosslinking: riboflavin concentration in corneal stroma exposed with and without epithelium. J Cataract Refract Surg 2009; 35: 893-899.

8 Mazzotta C, Balestrazzi A, Baiocchi S, Traversi C, Caporossi A. Stromal haze after combined riboflavin-UVA corneal collagen cross-linking in keratoconus: in vivo confocal microscopic evaluation. Clin Experiment Ophthalmol 2007; 35: 580-582.
9 Filippello M, Stagni E, O'Brart D. Transepithelial corneal collagen cross-linking: bilateral study. J Cataract Refract Surg 2012; 38(2): 283-291.

10 Zhang ZY, Zhang XR. Efficacy and safety of transepithelial corneal collagen crosslinking. J Cataract Refract Surg 2012; 38(7): 1304 author reply 1304-5.

11 Rechichi M, Daya S, Scorcia V, Meduri A, Scorcia G. Epithelial-disruption collagen crosslinking for keratoconus: one-year results. J Cataract Refract Surg 2013; 39(8): 1171-1178.

12 Samaras K, O'Brart DP, Doutch J, Hayes S, Marshall J, Meek KM. Effect of epithelial retention and removal on riboflavin absorption in porcine corneas. J Refract Surg 2009; 25(9): 771-775.

13 Sykakis E, Karim R, Evans JR, Bunce C, Amissah-Arthur KN, Patwary $\mathrm{S}$ et al. Corneal collagen cross-linking for treating keratoconus Cochrane Database Syst Rev, In press.

14 Raiskup F, Spoerl E. Corneal cross-linking with hypo-osmolar riboflavin solution in thin keratoconic corneas. Am J Ophthalmol 2011; 152(1): 28-32.

15 Rama P, Di Matteo F, Matuska S, Paganoni G, Spinelli A. Acanthamoeba keratitis with perforation after corneal crosslinking and bandage contact lens use. J Cataract Refract Surg 2009; 35(4): 788-791.

16 Kymionis GD, Portaliou DM, Bouzoukis DI, Suh LH, Pallikaris AI, Markomanolakis M et al. Herpetic keratitis with iritis after corneal crosslinking with riboflavin and ultraviolet A for keratoconus. J Cataract Refract Surg 2007; 33(11): 1982-1984. 